\title{
Musgos (Bryophyta) na Ilha Trambioca, Barcarena, PA, Brasil ${ }^{1}$
}

\author{
Adriana Paula da Silva Souza ${ }^{2,4}$ e Regina Célia Lobato Lisboa ${ }^{3}$
}

Recebido em 15/06/2004. Aceito em 03/12/2004

\begin{abstract}
RESUMO - (Musgos (Bryophyta) na Ilha Trambioca, Barcarena, PA, Brasil). Foi realizado o inventário dos musgos em diferentes ecossistemas da Ilha Trambioca, município de Barcarena, PA. Foram encontradas 40 espécies, pertencentes a 20 gêneros e 13 famílias. As famílias com maior número de espécies foram Calymperaceae (12 spp.), Sematophyllaceae (6 spp.) e Leucobryaceae (5 spp.), as quais destacaram-se também quanto ao número de indivíduos. As espécies mais comuns foram Sematophyllum subsimplex (Hedw.) Mitt, Calymperes erosum Müll. Hal., Pilosium chlorophyllum (Hornsch.) Müll. Hal., Taxithelium planum (Brid.) Mitt., Callicostella pallida (Hornsch.) Årgstr. e Trichosteleum papillosum (Hornsch.) A. Jaeger. Evidenciou-se maior diversidade de espécies na mata de terra firme, confirmando a preferência das briófitas por este ecossistema. Philonotis gracillima Ångstr. é citada como primeira referência para o Estado do Pará.
\end{abstract}

Palavras-chave: Briófitas, diversidade, Philonotis gracillima Ångstr., brioflora

ABSTRACT - (Mosses (Bryophyta) in Trambioca Island, Barcarena, Pará State, Brazil). This work presents a survey of mosses from different ecosystems of Trambioca Island, municipality of Barcarena, Pará State, Brazil. Forty taxa of mosses were found, belonging to 20 genera and 13 families. The families with highest number of species were Calymperaceae (12 spp.), Sematophyllaceae (6 spp.), and Leucobryaceae ( $5 \mathrm{spp}$.), which also presented the highest number of individuals. The most common species were Sematophyllum subsimplex (Hedw.) Mitt, Calymperes erosum Müll. Hal., Pilosium chlorophyllum (Hornsch.) Müll. Hal., Callicostella pallida (Hornsch.) Årgstr., and Trichosteleum papillosum (Honrsch.) A. Jaeger. It was observed a higher richness of species in the primary low land rainforest, confirming the preference of bryophytes for this ecosystem. Philonotis gracillima Ångstr. is mentioned as the first report to Pará State.

Key words : Bryophytes, diversity, Philonotis gracillima Ångstr., bryoflora

\section{Introdução}

O município de Barcarena pertence à Mesoregião metropolitana de Belém. É limitado em grande parte (norte e oeste) pela Baía de Marajó e recortado por inúmeros rios, furos e igarapés, caracterizando-se como área de estuário. Apresenta condições naturais específicas de níveis topográficos pouco elevados, sobretudo nas ilhas sujeitas, em parte, às inundações.

Os estudos de florística no município de Barcarena referem-se somente a levantamento botânico de espécies usadas com fins terapêuticos (Amorozo \& Gély 1988), além de um estudo de macrófitas aquáticas (Lins et al. 1989) e um levantamento etnobotânico (Amorozo 1997), sendo que este complementa a listagem elaborada anteriormente por Amorozo \& Gély (1988). Em relação à brioflora, há somente coletas feitas por botânicos do Museu Paraense Emílio Goeldi, estando o material depositado no herbário desta instituição (MG).
Recentemente foi realizado pela equipe do Museu Paraense Emílio Goeldi levantamento botânico da vegetação de Barcarena, com análises qualitativas e quantitativas, resultando na elaboração de um relatório (Amaral et al. 2002). Neste relatório, os resultados referentes à flora fanerogâmica são bastante expressivos, mas trazem poucas informações sobre as espécies de briófitas, havendo necessidade de estudos mais completos. Desta forma, este trabalho tem como objetivo caracterizar a diversidade de musgos desta localidade, complementando os resultados encontrados para o município.

\section{Material e métodos}

Área de estudo - A ilha de Trambioca localiza-se no município de Barcarena, entre as coordenadas geográficas de $01^{\circ} 23^{\prime}-01^{\circ} 31^{\prime} \mathrm{S}$ e $48^{\circ} 36^{\prime}-48^{\circ} 42^{\prime} \mathrm{W}$. Apresenta extensão aproximada de $123,5 \mathrm{~km}^{2}$, sendo banhada pelo rio Mucuruçá, furo do Arrozal, rio

\footnotetext{
Parte da dissertação de Mestrado da primeira Autora

Universidade Federal Rural da Amazônia, Departamento de Botânica, Museu Paraense Emílio Goeldi (regina@museu-goeldi.br)

Museu Paraense Emílio Goeldi, C. Postal 399, CEP 66040-170, Belém, PA, Brasil

Autor para correspondência: apsouza@museu-goeldi.br
} 
Carnapijó e baía do Marajó. Segundo a classificação de Köppen, o clima da região é do tipo Am, caracterizando-se como clima quente equatorial, com temperatura média anual de $27^{\circ} \mathrm{C}$ e com amplitude térmica mínima. A precipitação anual é superior a $2.500 \mathrm{~mm}$, com estação chuvosa entre janeiro a junho e mais seca nos últimos meses do ano. A vegetação representada na ilha é composta, basicamente, por matas primárias de terra firme, capoeiras, matas de várzea e campinas arenosas e praias de água doce (Amaral et al. 2002).

Coleta e identificação - A amostras foram coletadas, nas diferentes formações vegetais da ilha Trambioca, entre novembro/2001 e março/2002. O total de 166 amostras de briófitas foram estudadas. As amostras foram retiradas de diferentes tipos de substratos, sendo as espécies classificadas segundo Robbins (1952) em: corticícolas - troncos e ramos de árvores vivas; epíxilas - ramos e troncos caídos e em decomposição; rupícolas - superfície rochosa; terrestres - superfície do solo ou litter. Algumas amostras foram coletadas sobre cupinzeiro. As identificações basearam-se nas publicações de Florschütz (1964), Griffin III (1979), Crum \& Anderson (1981), Florschütz-De Waard (1986), O. Yano (dados não publicados), Lisboa (1993), Ireland \& Buck (1994), Churchill \& Linares (1995), Florschütz-De Waard (1996), Florschütz-De Waard \& Veling (1996), Veling (1996) e Zielman (1996).

\section{Resultados e discussão}

$\mathrm{Na}$ ilha Trambioca foram registrados 40 táxons de musgos, distribuídos em 20 gêneros e 13 famílias (Tab. 1). As famílias mais representativas em número de espécies foram Calymperaceae, Sematophyllaceae e Leucobryaceae, as quais são de ocorrência comum em toda a região amazônica, de acordo com os trabalhos de Lisboa \& Maciel (1994), Lisboa \& Nazaré (1997), Lisboa et al. (1998), Lisboa et al. (1999) e Santos \& Lisboa (2003).

O ecossistema de floresta de terra firme apresentou o maior número de espécimes coletados, seguido das áreas de capoeira, mata de várzea, praia de rio e campina (Tab. 1).

A maior diversidade e a ocorrência de espécies para a mata de terra firme, como já relatado nos trabalhos de Lisboa \& Nazaré (1997), Santos \& Lisboa (2003) e no levantamento sobre a diversidade das briófitas na Amazônia Brasileira (Lisboa 2003), confirmam a preferência das espécies por esse ecossistema, o que pode ser explicado pelo conjunto de fatores que propiciam melhores condições ao desenvolvimento de briófitas, tais como maior número de substratos, umidade, forófitos mais bem estruturados, menor exposição à dessecação, etc.

A mata de capoeira apresentou riqueza de 21 espécies. Segundo Gradstein (1992), as florestas secundárias com maior diversidade florística podem reter de 50-70\% das espécies de briófitas das florestas não perturbadas, ressaltando, portanto, a importância da conservação deste ecossistema. A baixa riqueza observada nos demais ecossistemas deve-se a condições pouco adequadas para o estabelecimento deste grupo vegetal, como por exemplo: maior intensidade de luz e calor, baixa umidade, menor disponibilidade de substratos.

Quanto ao substrato utilizado, é evidenciado grande número de musgos epíxilos, representados principalmente por Pilosium chlorophyllum, Callicostella pallida e Leucomium strumosum, que ocorreram com frequiência associados entre si e com outras espécies, recobrindo abundantemente troncos caídos e/ou apodrecidos, fato também observado por Lisboa \& Ilkiu-Borges (2004).

Por outro lado, Calymperes lonchophyllum, Octoblepharum pulvinatum e Phyllodrepanium falcifolium foram coletadas crescendo isoladamente sobre ramos de árvores vivas. Não foram evidenciados musgos epífilos na área estudada.

Sematophyllum subsimplex, Taxithelium planum, Trichosteleum papillosum e Calymperes erosum estão entre as espécies mais comuns, da mesma forma que Callicostella pallida e Pilosium chlorophyllum. Todas essas espécies são muito comuns na região de mata amazônica (Lisboa \& Maciel 1994; Lisboa 2003).

Comparando a diversidade de musgos encontrada em Barcarena, onde 43 táxons foram identificados, com a da ilha Trambioca (40 táxons), observam-se 31 espécies comuns às duas áreas, enquanto Calymperes pallidum, Syrrhopodon simmondsii, Campylopus surinamensis, Fissidens prionodes, Fissidens scariosus, Ectropothecium leptochaeton, Groutiela tomentosa, Lepidopilum surinamense, Pilotrichum bipinnatum, Henicodium geniculatum, Meiothecium boryanum e Sematophyllum subpinnatum foram coletadas apenas em Barcarena, e Philonotis gracillima, Syrrhopodon hornschuchii, Syrrhopodon parasiticus, Fissidens guianensis var. guianensis, Fissidens radicans, Phyllodrepanium falcifolium, Pilotrichum evanescens, Hyophila involuta e 
Tabela 1. Substratos e ecossistemas de ocorrência dos musgos da ilha Trambioca, município de Barcarena, Pará, Brasil. Substratos: Co - Corticícola; Ep - Epíxila; Ru - Rupícola; Cup - Cupinzeiro. Ecossistemas: Mt - Mata de terra firme; Pr - Praia; Cp - Capoeira; Ca - Campina; Va - Várzea. Os números indicam o registro de cada táxon em cada ecossistema e nos substratos.

\begin{tabular}{|c|c|c|c|c|c|c|c|c|c|}
\hline \multirow[b]{2}{*}{ Família/Espécie } & \multicolumn{4}{|c|}{ Substrato } & \multicolumn{5}{|c|}{ Ecossistema } \\
\hline & $\mathrm{Co}$ & Ep & $\mathrm{Ru}$ & Cup & $\mathrm{Mt}$ & $\operatorname{Pr}$ & $\mathrm{Cp}$ & $\mathrm{Ca}$ & Va \\
\hline \multicolumn{10}{|l|}{ BARTRAMIACEAE } \\
\hline Philonotis gracillima Ångstr. & - & - & 1 & - & - & - & 1 & - & - \\
\hline \multicolumn{10}{|l|}{ CALYMPERACEAE } \\
\hline Calymperes afzelii Sw. & - & 1 & - & - & - & 1 & - & - & - \\
\hline C. erosum Müll. Hal. & 4 & 8 & - & - & 7 & - & 4 & 1 & - \\
\hline C. levyanum Besch. & 2 & 1 & - & - & 2 & - & - & - & 1 \\
\hline C. lonchophyllum Schwägr. & 7 & - & - & - & 4 & - & 3 & - & - \\
\hline C. palisotii Schwägr. & 1 & 3 & - & - & - & 4 & - & - & - \\
\hline $\begin{array}{l}\text { Syrrhopodon africanus (Mitt.) Paris } \\
\text { subsp. graminicola (R.S. Williams) W.D. Reese }\end{array}$ & 1 & 1 & - & - & - & - & 1 & 1 & - \\
\hline S. cryptocarpos Dozy \& Molk. & 2 & - & - & - & 1 & - & 1 & - & - \\
\hline S. hornschuchii C. Mart. & 1 & - & - & - & 1 & - & - & - & - \\
\hline S. incompletus Schwägr. var. incompletus & 2 & - & - & - & 2 & - & - & - & - \\
\hline S. ligulatus Mont. & 1 & 1 & - & - & 1 & - & 1 & - & - \\
\hline S. parasiticus (Brid.) Besch. & 1 & - & - & - & - & - & 1 & - & - \\
\hline S. rigidus Hook. \& Grev. & 1 & - & - & - & 1 & - & - & - & - \\
\hline \multicolumn{10}{|l|}{ FISSIDENTACEAE } \\
\hline Fissidens elegans Brid. & 3 & 3 & 1 & - & 2 & - & 3 & - & 2 \\
\hline F. guianensis Mont. var. guianensis & - & 1 & - & - & 1 & - & - & - & - \\
\hline F. pellucidus Hornsch. var. pellucidus & - & 4 & - & - & 4 & - & - & - & - \\
\hline F. radicans Mont. & - & - & 2 & - & - & - & 2 & - & - \\
\hline \multicolumn{10}{|l|}{ HYPNACEAE } \\
\hline Isopterygium acutifolium Ireland & - & 1 & - & - & - & 1 & - & - & - \\
\hline I. tenerum (Sw.) Mitt. & 1 & - & - & - & - & 1 & - & - & - \\
\hline Vesicularia vesicularis (Schwägr.) Broth. & - & 2 & - & - & 2 & - & - & - & - \\
\hline \multicolumn{10}{|l|}{ LEUCOBRYACEAE } \\
\hline Leucobryum albidum (Brid. ex P. Beauv.) Lindb. & 1 & 1 & - & 1 & 1 & - & 2 & - & - \\
\hline L. martianum (Hornsch.) Hampe & - & 3 & - & 1 & 2 & - & 2 & - & - \\
\hline Octoblepharum albidum Hedw. var. albidum & 2 & 2 & - & - & 2 & - & 1 & - & 1 \\
\hline O. albidum Hedw. var. violascens Müll. Hal. & 1 & - & - & - & 1 & - & - & - & - \\
\hline O. pulvinatum (Dozy \& Molk.) Mitt. & 7 & - & - & - & 6 & - & 1 & - & - \\
\hline \multicolumn{10}{|l|}{ LEUCOMIACEAE } \\
\hline Leucomium strumosum (Hornsch.) Mitt. & 1 & 6 & - & - & 5 & - & - & - & 2 \\
\hline \multicolumn{10}{|l|}{ NECKERACEAE } \\
\hline Neckeropsis undulata (Hedw.) Reichardt & 1 & - & - & - & - & - & - & - & 1 \\
\hline \multicolumn{10}{|l|}{ PHYLLODREPANIACEAE } \\
\hline Phyllodrepanium falcifolium (Schwägr.) Crosby & 7 & - & - & - & 7 & - & - & - & - \\
\hline \multicolumn{10}{|l|}{ PILOTRICHACEAE (CALLICOSTACEAE) } \\
\hline Callicostella pallida (Hornsch.) Ångstr. & 1 & 9 & - & - & 7 & - & 1 & - & 2 \\
\hline Pilotrichum evanescens (Müll. Hal.) Crosby & 1 & - & - & - & - & - & - & - & 1 \\
\hline \multicolumn{10}{|l|}{ POTTIACEAE } \\
\hline Hyophila involuta (Hook.) A. Jaeger & 1 & - & - & - & 1 & - & - & - & - \\
\hline Hyophiladelphus agrarius (Hedw.) R.H. Zander & - & - & 2 & - & - & - & 2 & - & - \\
\hline \multicolumn{10}{|l|}{ SEMATOPHYLLACEAE } \\
\hline Sematophyllum subsimplex (Hedw.) Mitt. & 8 & 13 & - & - & 14 & - & 6 & - & 1 \\
\hline Taxithelium planum (Brid.) Mitt. & 5 & 6 & & 1 & 7 & 3 & 2 & & \\
\hline T. pluripunctatum (Renauld \& Cardot) W.R. Buck & 4 & - & - & - & 2 & - & 2 & - & - \\
\hline Trichosteleum bolivarense H. Rob. & - & 3 & - & - & 2 & - & - & - & 1 \\
\hline T. hornschuchii (Hampe) A. Jaeger & - & 3 & - & - & 2 & - & - & - & 1 \\
\hline T. papillosum(Hornsch.) A. Jaeger & 3 & 5 & & 1 & 4 & & 4 & & 1 \\
\hline \multicolumn{10}{|l|}{ STEREOPHYLLACEAE } \\
\hline Pilosium chlorophyllum (Hornsch.) Müll. Hal. & 3 & 7 & - & 2 & 9 & - & 3 & - & - \\
\hline THUIDIACEAE & & & & & & & & & \\
\hline Cyrto-hypnum scabrosulum (Mitt.) W.R. Buck \& H.A. Crum & 2 & 2 & 1 & - & 3 & - & 1 & - & 1 \\
\hline Total & 75 & 86 & 7 & 6 & 103 & 10 & 44 & 2 & 15 \\
\hline
\end{tabular}


Hyophiladelphus agrarius ocorreram apenas na ilha Trambioca.

A diferença na diversidade de musgos em áreas geográficas tão próximas, separadas apenas por um furo de poucos metros de largura, evidencia a necessidade de estudos mais criteriosos, para alcançar efetivo conhecimento da biodiversidade.

Durante o presente estudo evidenciou-se ainda que Philonotis gracillima é considerada como a primeira ocorrência para o Estado do Pará, para a qual são apresentados descrição, ilustração e comentários sobre as relações desta com outras espécies do gênero.
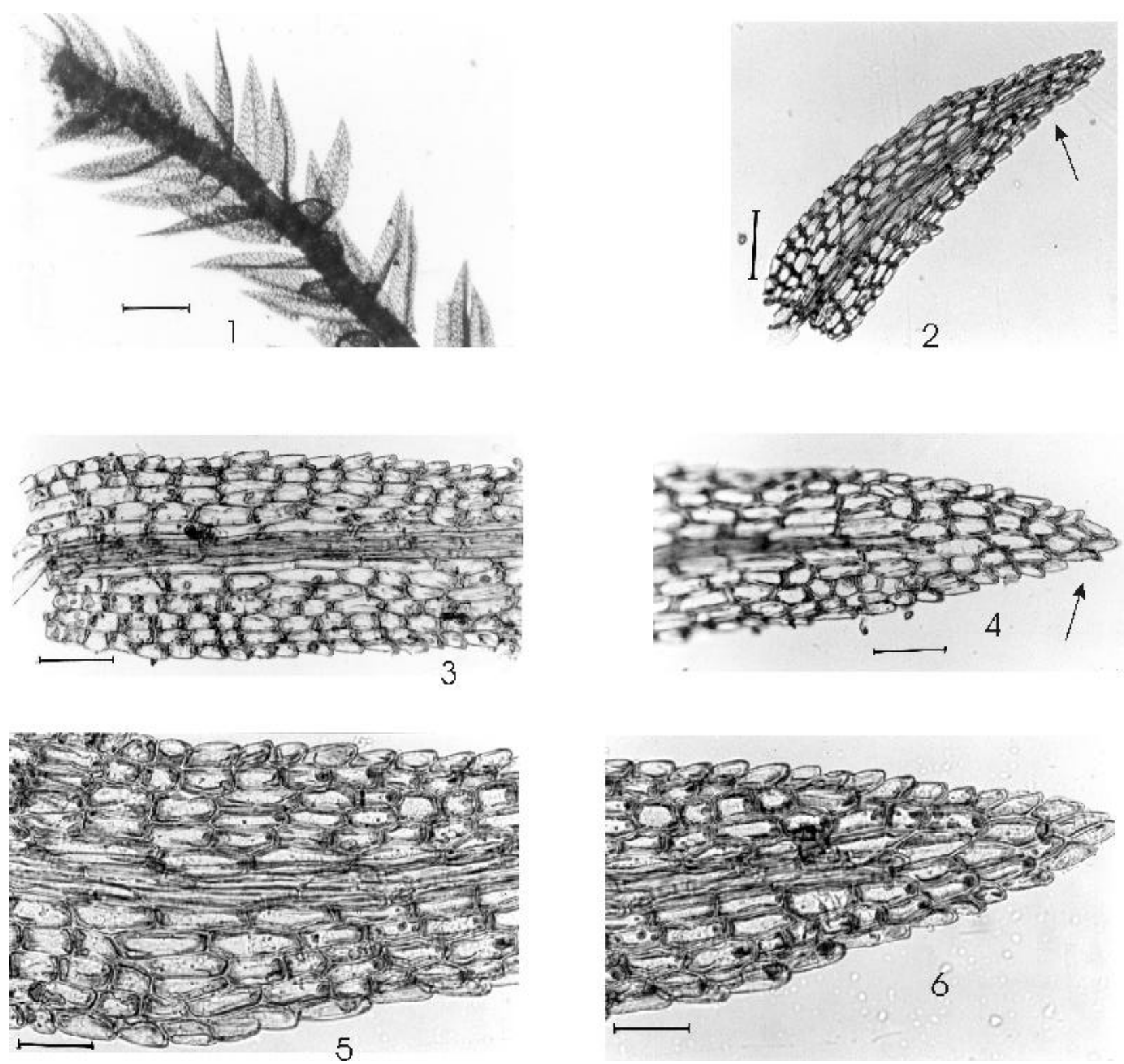

Família Bartramiaceae Schwägr.

Philonotis gracillima Ångstr. Öfversigt af Förhandlingar: Kongl. Svenska Vetenskaps-Akademien 33(4): 17. 1876. Tipo: Brasil, Regnell 38 (S-PA n.v., $\mathrm{BM}$ n.v.).

Fig. 1-6.

Gametófitos verdes, formando tufos densos ou frouxos, pequenos e delgados (4-6 mm), tomentosos na face abaxial; caulídios castanho-claros, raramente ramificados; filídios em várias fileiras, espaçados, nunca ou raramente juntos, secundos quando secos,
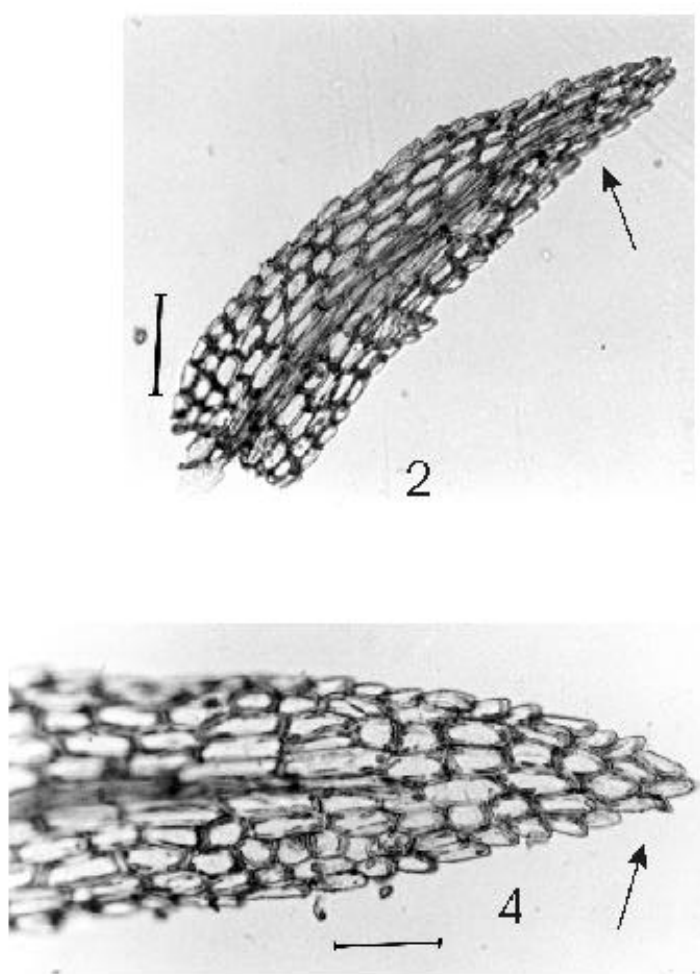

Figuras 1-6. Philonotis gracillima Ångstr. 1. Aspecto do gametófito. 2. Filídio, detalhe da margem serreada. 3. Células medianas mais largas e longas prox. da costa. 4. Ápice do filídio, com papilas terminais bem evidentes. 5. Células marginais curto-retangulares. 6 . Ápice mostrando costa subpercurrente (Fotos: A.P.S. Souza, 2004; R. Lisboa 7123); Escalas $1=550 \mu \mathrm{m} ; 2=140 \mu \mathrm{m} ; 3$ e $4=100 \mu \mathrm{m} ; 5 \mathrm{e}$ $6=60 \mu \mathrm{m}$.

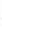


expandidos quando úmidos, lanceolados, ápice obtuso; costa subpercurrente, terminando 3-4 células abaixo do ápice; margem serreada; células do filídio unipapilosas, papilas terminais; células basais quadráticas; células medianas retangulares, mais alongadas e largas nas proximidades da costa; células marginais curto-retangulares. Esporófito não observado.

Material examinado: BRASIL. Pará: Barcarena, ilha Trambioca, na base de muro de ponte sobre manilha da estrada, 17/XI/2001, R. Lisboa 7123 (MG).

Distribuição geográfica: citada para os Estados de MG, PR, RJ, SC, SP (Yano 1981); BA (Yano 1989); ES (como P. uncinata (Schwägr.) Brid. var. gracillima (Ångstr.) Florsch. por Yano, 1995) e AM (Churchill 1998).

Hábitat: encontrada preferencialmente em locais úmidos, como rupícola ou terrestre.

Comentários: o gênero Philonotis tem sido alvo de constantes especulações com relação à identificação de algumas de suas espécies. Florschütz (1964), em um estudo sobre o complexo de espécies de Philonotis gracillima, considerou que Philonotis uncinata, P. gracillima e Philonotis glaucescens, representariam apenas variedades de uma mesma espécie, baseando-se na morfologia e disposição dos filídios, como exposto a seguir: Philonotis uncinata var. uncinata, filídios falcados no topo dos caulídios, com ápice agudo e costa excurrente; Philonotis uncinata var. gracillima (Ångstr.) Florsch., filídios não falcados, ápice obtuso ou arredondado e costa terminando bem abaixo do ápice (subpercurrente); Philonotis uncinata var. glaucescens (Hornsch.) Florsch., filídios não falcados, com ápice agudo e costa percurrente.

No entanto, Crum \& Anderson (1981) e Churchill \& Linares (1995) consideram estas características suficientes para mantê-las como espécies separadas, devido a $P$. glaucescens apresentar normalmente filídios com ápice agudo, um pouco mais longos e costa percurrente. Em vista do exposto, P. gracillima é considerada uma espécie válida, sendo necessário novas evidências antes de considerá-la como sinônimo de $P$. glaucescens.

No presente estudo observou-se que Philonotis gracillima apresentou os filídios com ápice obtuso ou arredondado e costa terminando bem abaixo do ápice (subpercurrente) em todos os filídios e, conforme sugerido por Crum \& Anderson (1981) e Churchill \& Linares (1995), este táxon foi considerado no nível de espécie, configurando-se a primeira ocorrência para o Estado do Pará.

\section{Agradecimentos}

As autoras agradecem à Companhia Texaco, pelo apoio financeiro para as coletas do material botânico.

\section{Referências bibliográficas}

Amaral, D.D.; Bastos, M.N.; Silva, A.S.L.; Oliveira, J.; Lisboa, L.R.C.; Rosário, C.S.; Gomes, A.; Silva, C.A. \& Aguiar, J. 2002. Inventário da Flora da região de Barcarena, Pará. Relatório Final. Belém, Ministério da Ciência e Tecnologia, Museu Paraense Emílio Goeldi.

Amorozo, M.C.M. \& Gély, A. 1988. Uso de plantas medicinais por caboclos do Baixo Amazonas, Barcarena, PA, Brasil. Boletim do Museu Paraense Emílio Goeldi, série Botânica 4(1): 47-131.

Amorozo, M.C.M. 1997. Algumas notas adicionais sobre o emprego de plantas e outros produtos com fins terapêuticos pela população cabocla do município de Barcarena, PA, Brasil. Boletim do Museu Paraense Emílio Goeldi, série Botânica 13(2): 191-213.

Churchill, S.P. 1998. Catalog of Amazonian Mosses. The Journal of the Hattori Botanical Laboratory 85: 191-238.

Churchill, S.P. \& Linares, C.E.L. 1995. Prodomus Bryologiae Novo-Granatensis: Introduccion a la Flora de Musgos de Colombia. Biblioteca José Jeronimo Triana 12: 105-134.

Crum, H.A. \& Anderson, L.E. 1981. Mosses of Easten North America vol 1. New York, Columbia University Press.

Florschütz, P.A. 1964. The Mosses of Suriname. Part. I. Pp. 1-271. In: J. Lanjouw (ed.). Flora of Suriname. Leiden, E.J. Brill.

Florschütz-De Waard, J. 1986. Hookeriaceae. Musci. II. Pp. 289-350. In: A.L. Stoffers \& J.C. Lindeman (eds.). Flora of Suriname. Leiden, E.J. Brill.

Florschütz-De Waard, J. 1996. Sematophyllaceae. Musci III. Pp. 384-438. In: A.R.A. Görts-Van Rijn (ed.). Flora of the Guianas. Series C: Bryophytes.

Florschütz-De Waard, J. \& Veling, K. 1996. Hypnaceae. Musci III. Fascicle 1. Pp. 439-462. In: A.R.A. GörtsVan Rijn (ed.). Flora of the Guianas. Series C: Bryophytes.

Gradstein, S.R. 1992. The vanishing tropical rain forest as an environment for bryophytes and lichens. Pp. 232-256. In: J.W. Bates \& A.M. Farmer (eds.). Bryophytes and lichens in a Changing Environment. Oxford, Clarendon Press.

Griffin III, D. 1979. Guia preliminar para as briófitas freqüentes em Manaus e adjacências. Acta Amazônica 93): 1-67. (Suplemento).

Ireland, R.R. \& Buck, W.R. 1994. Stereophyllaceae. Flora Neotropica, Monograph 65: 1-51. 
Lins, A.L.F.A.; Potiguara, R.C.V.; Rosa, N.A. \& Ribeiro, I.C. 1989. Macrófitos aquáticos de uma área de Barcarena, Pará, Brasil. Boletim do Museu Paraense Emílio Goeldi, série Botânica 5(2): 135-144.

Lisboa, R.C.L. 1993. Musgos Acrocárpicos do Estado de Rondônia. Coleção Adolpho Ducke. Belém, Museu Paraense Emílio Goeldi.

Lisboa, R.C.L. 2003. Diversidade das Briófitas do Estado do Pará. Pp. 57-60. In: M.A. Jardim; M.N.C. Bastos \& J.U.M. Santos (eds.). Desafios da Botânica no Novo Milênio: Inventário, Sistematização e Conservação da Diversidade vegetal. $54^{\circ}$ Congresso Nacional de Botânica.

Lisboa, R.C.L. \& Ilkiu-Borges, A.L. 2004. Uma nova avaliação da Brioflora da Reserva Mocambo, Belém (PA). In: J.I. Gomes (org.). História Natural e Biologia da área de Pesquisa Ecológica do Guamá-Apeg.

Lisboa, R.C.L. \& Maciel, U.N. 1994. Musgos da Ilha de Marajó-I-Afuá. Boletim do Museu Paraense Emílio Goeldi, série Botânica 10(1): 43-55.

Lisboa, R.C.L. \& Nazaré, J.M.M. 1997. A Flora Briológica. Pp. 223-235. In: P.L.B. Lisboa (org.). Caxiuanã. Belém, $\mathrm{CNPq} / \mathrm{MPEG}$.

Lisboa, R.C.L.; Muniz, A.C.M. \& Maciel, U.N. 1998. Musgos da Ilha de Marajó-III- Chaves (Pará). Boletim do Museu Paraense Emílio Goeldi, série Botânica 14(2): 117-125.
Lisboa, R.C.L.; Lima, M.J.L. \& Maciel, U.N. 1999. Musgos da Ilha de Marajó - II - Município de Anajás, Pará, Brasil. Acta Amazônica 29(2): 201-206.

Robbins, R.G. 1952. Bryophyta Ecology of a dune area in New Zealand. Vegetation. Acta Geobotanica 4: 1-31.

Santos, R.C. \& Lisboa, R.C.L. 2003. Contribuição ao Estudo dos Musgos (Bryophyta) no Nordeste Paraense, Zona Bragantina, Microrregião do Salgado e Município de Viseu, Pará. Acta Amazônica 33(3): 415-422.

Veling, K. 1996. Leucomiaceae. Musci III. Fascicle 1. Pp. 365-370. In: A.R.A. Görts-Van Rijn (ed.). Flora of the Guianas. Series C: Bryophytes.

Yano, O. 1981. A Checklist of Brazilian mosses. The Journal of the Hattori Botanical Laboratory 69: 147-170.

Yano, O. 1989. An additional checklist of Brazilian bryophytes. The Journal of the Hattori Botanical Laboratory 66: 371-434.

Yano, O. 1995. A new additional annotated checklist of Brazilian bryophytes. The Journal of the Hattori Botanical Laboratory 78: 137-182.

Zielman, H. R. 1996. Thuidiaceae. Musci III. Fascicle 1. Pp. 371-383. In: A.R.A. Görts-Van Rijn (ed.). Flora of the Guianas. Series C: Bryophytes. 